\title{
Identificación rápida de micobacterias no tuberculosas en Cuba por las técnicas "GenoType Mycobacterium CM/AS»
}

\section{Rapid identification of nontuberculous mycobacteria in Cuba by the "GenoType Mycobacterium CM/AS» techniques}

\author{
Misleidis Sardiñas-Aragón,* Lilian María Mederos-Cuervo,* Grechen García-León,* \\ María Rosarys-Martínez, ${ }^{*}$ Dihadenys Lemus-Molina,* Raúl Díaz-Rodríguez*
}

*Laboratorio Nacional de Referencia e Investigaciones de Tuberculosis, Lepra y Micobacterias. Instituto de Medicina Tropical «Pedro Kourí». La Habana, Cuba.

\begin{abstract}
RESUMEN. Introducción: En los últimos años las infecciones ocasionadas por micobacterias no tuberculosas han tenido un incremento notable en varias regiones del mundo, asociado fundamentalmente a infecciones respiratorias en individuos inmunocomprometidos. La identificación de micobacterias no tuberculosas es engorrosa y puede demorar varias semanas. Objetivo: Aplicar las técnicas moleculares GenoType Mycobacterium CM/AS para la identificación de micobacterias no tuberculosas en Cuba y comparar con los resultados de la prueba convencional. Material y métodos: Se utilizaron 73 aislados de micobacterias no tuberculosas procedentes de muestras pulmonares recibidas de unidades de salud ubicadas en varias provincias de Cuba en el período abril 2017-diciembre 2018. Para la identificación de especies se utilizaron técnicas convencionales y las técnicas moleculares GenoType CM/AS. Resultados: Las especies identificadas más predominantes fueron las pertenecientes al complejo Mycobacterium avium-intracellulare (46.6\%) y Mycobacterium fortuitum (31.5\%). Se hallaron cuatro aislados de M. simiae, especie poco frecuente en Cuba. Al comparar los resultados de ambas técnicas se obtuvo una concordancia de $91.3 \%$. Conclusión: Las técnicas GenoType Mycobacterium CM/AS demostraron ser herramientas útiles en la identificación rápida de micobacterias en el Laboratorio de Referencia, brindando resultados precisos en menor tiempo, logrando acortar significativamente el tiempo diagnóstico. Las especies más frecuentes en Cuba siguen siendo el complejo Mycobacterium avium-intracellulare y Mycobacterium fortuitum.
\end{abstract}

Palabras clave: Micobacterias no tuberculosas, identificación de especie, GenoType Mycobacterium CM, GenoType Mycobacterium AS.
ABSTRACT. Introduction: In recent years, infections caused by nontuberculous mycobacteria (NTM) have had a remarkable increase in several regions of the world, mainly associated with respiratory infections in immunocompromised individuals. The identification of MNT is cumbersome and may take several weeks. Objective: Apply the molecular techniques GenoType Mycobacterium CM/AS for the identification of MNT in Cuba, and compare with the results of conventional tests. Material and methods: We used 73 MNT isolates from lung samples received from health units located in several provinces of Cuba in the period April 2017 - December 2018. For the identification of species, conventional techniques and molecular techniques were used GenoType CM/AS. Results: The most predominant identified species were those belonging to the $M$. avium-intracellulare complex $(46.6 \%)$ and the Mycobacterium fortuitum (31.5\%). Four isolates of M. simiae, a rare species in Cuba, were found. When comparing the results of both techniques, a concordance of $91.3 \%$ was obtained. Conclusion: GenoType Mycobacterium CM/AS techniques proved to be useful tools in the rapid identification of mycobacteria in the Reference Laboratory, providing accurate results in less time, significantly shortening the diagnostic time. The most frequent species in Cuba continue to be the M. avium-intracellulare complex and the Mycobacterium fortuitum.

Keywords: Non tuberculous mycobacteria (NTM), species identification, GenoType Mycobacterium CM, GenoType Mycobacterium AS.

\section{Correspondencia:}

\section{Lic. Misleidis Sardiñas Aragón}

Laboratorio Nacional de Referencia e Investigaciones de Tuberculosis, Lepra y Micobacterias. Instituto de Medicina Tropical «Pedro Kourí». Correo electrónico: misle@ipk.sld.cu/misleidiss@infomed.sld.cu

Trabajo recibido: 30-I-2019; aceptado: 18-II-2019.

\section{INTRODUCCIÓN}

Las micobacterias se encuentran distribuidas ampliamente en el medio ambiente. Hasta ahora se han descrito más de 150 especies, alguna de ellas son saprófitas y otras son patógenas o se comportan como patógenas oportunistas. ${ }^{1}$ 
Las micobacterias no tuberculosas (MNT) son aquellas especies que no pertenecen al complejo Mycobacterium tuberculosis (MTB) ni al complejo Mycobacterium leprae. Éstas son agentes causales de severas patologías y afectan pulmones (preferiblemente), glándulas linfoides, piel, heridas y huesos. Las principales especies que aparecen como causa de infección en humanos son el complejo $M$. avium-intracellulare, M. kansasii, M. szulgai, M. marinum y el complejo M. fortuitum-chelonae. ${ }^{2}$

Las infecciones ocasionadas por las MNT han tenido un incremento en los últimos años y se asocian con una elevada morbimortalidad, sobre todo en individuos con inmunidad local o sistémica alterada, tal como los pacientes infectados con el virus de inmunodeficiencia humana (VIH). También se han desarrollado técnicas diagnósticas con mayor sensibilidad y especificidad que han permitido mejorar la identificación de este tipo de infección. ${ }^{3-5}$

La identificación tradicional de las MNT se basa en el análisis de las características fenotípicas, biológicas y bioquímicas tales como: morfología, velocidad de crecimiento, producción de pigmento, actividad enzimática y la utilización de sustratos. Muchas de estas técnicas requieren de varias semanas para obtener sus resultados, y algunas no permiten la diferenciación de miembros pertenecientes a un mismo complejo. ${ }^{6}$

Actualmente, se han desarrollado técnicas de hibridación reversa y tecnología de sonda en línea, como el ensayo GenoType Mycobacterium CM (Hain Lifescience, Nehren, Alemania). Esta es una nueva herramienta para la identificación de MNT, la prueba se basa en la amplificación del ácido desoxirribonucleico (ADN) de una región del gen que codifica para el ácido ribonucleico ribosómico (ARNr) 23S. Esta técnica permite la diferenciación rápida y confiable de las 24 especies más aisladas en humanos, ya que cada una de éstas genera un patrón específico lográndose una identificación rápida y, por tanto, la aplicación oportuna del tratamiento adecuado. La empresa Hain Lifescience dispone también del ensayo GenoType Mycobacterium AS para la identificación de otras MNT menos frecuentes, las cuales no son detectadas por el sistema GenoType Mycobacterium CM. . $^{7-9}$

El objetivo de este estudio fue aplicar por primera vez en Cuba las técnicas moleculares GenoType Mycobacterium CM/AS para la identificación de MNT con vistas a lograr un acortamiento en el tiempo diagnóstico, y comparar sus resultados con los obtenidos por las pruebas convencionales.

\section{MATERIALES Y MÉTODOS}

Se realizó un estudio descriptivo donde se introdujeron y evaluaron dos nuevas pruebas diagnósticas moleculares para la identificación de MNT. El presente trabajo se hizo en el período comprendido entre abril 2017 a diciembre 2018, en el Laboratorio Nacional de Referencia e Investigaciones de Tuberculosis, Lepra y Micobacterias (LNRI-TBLM) del Instituto de Medicina Tropical «Pedro Kourí» (IPK), La Habana, Cuba.

Se utilizaron 12 cepas de referencia para la estandarización de las técnicas moleculares, y se identificaron 73 aislados de MNT de muestras pulmonares de pacientes recibidas en el LNRI-TBLM-IPK desde la red nacional de laboratorios de TB del país.

Todos los aislados fueron subcultivados en medio Löwenstein-Jensen (L-J) y se incubaron a $37{ }^{\circ} \mathrm{C}$ durante 3-4 semanas. Después, se hicieron las pruebas fenotípicas y bioquímicas establecidas. La clasificación fenotípica se realizó según Runyon (1959). ${ }^{10}$ Ésta se basa en la determinación de la velocidad de crecimiento y producción de pigmentos. Se incluyeron también pruebas de identificación, como termoestabilidad de la enzima catalasa a $68^{\circ} \mathrm{C}$, reducción de nitratos a nitritos, ureasa, hidrólisis de tween 80 , tolerancia al cloruro de sodio 5\%, detección de la actividad de las enzimas pirazinamidasa y arilsulfatasa, toma de hierro y reducción de telurito de potasio. ${ }^{11}$

Los ensayos GenoType Mycobacterium CM y GenoType Mycobacterium AS (Hain Lifescience, Nehren, Alemania) se realizaron siguiendo las instrucciones del fabricante., Primero se evaluaron con las cepas de referencia y posteriormente los 73 aislados.

Para la extracción de ADN se utilizó el sistema comercial Genolyze (Hain Lifescience). En la amplificación de ADN de ambos ensayos se empleó un termociclador SpeedCycler ${ }^{2}$ (Analytik Jenna, Berlin, Alemania). Como control positivo se utilizó el suministrado por el sistema comercial y como control negativo se emplearon $5 \mu$ de agua de calidad de biología molecular. La hibridación se llevó a cabo en la plataforma de incubación en un agitador con temperatura semiautomatizado Twincubator (Hain Lifescience).

\section{RESULTADOS}

En la tabla 1 se muestran los resultados de la identificación de las 12 cepas de referencia utilizadas en las pruebas moleculares. Como se puede observar, nueve de ellas se identificaron correctamente con el sistema GenoType Mycobacterium CM, al hibridar las bandas designadas para cada especie. Mientras que en las otras tres el patrón de bandas obtenidas sólo permitió identificar hasta género Mycobacterium. ${ }^{8}$ Por tanto, con el ensayo GenoType Мyсоbacterium AS se identificaron correctamente las tres cepas antes mencionadas.

En la figura 1 se muestran los resultados obtenidos de la identificación en especies de los 73 aislados estudiados aplicando el método convencional. Las especies con mayor porcentaje de aislamiento fueron complejo M. aviumintracellulare (MAl) 34 aislados (46.6\%) y M. fortuitum 23 aislados (31.5\%). 
Neumol Cir Torax. 2019; 78 (3): 290-295

Tabla 1: Resultados de la identificación de las cepas de referencia por las técnicas GenoType Mycobacterium CM y GenoType Mycobacterium AS.

\begin{tabular}{|c|c|c|c|c|c|}
\hline Cepas de referencia & No & \multicolumn{2}{|c|}{ GenoType Mycobacterium CM } & \multicolumn{2}{|c|}{ GenoType Mycobacterium AS } \\
\hline M. fortuitum & 4 & $1,2,3,7,14$ & M. fortuitum & NR & NR \\
\hline M. intracellulare & 1 & $1,2,3,9$ & M. intracellulare & NR & NR \\
\hline M. tuberculosis & 1 & $1,2,3,10,16$ & M. tuberculosis & NR & NR \\
\hline M. gordonae & 1 & $1,2,3,8,10$ & M. gordonae & NR & NR \\
\hline M. simiae & 1 & $1,2,3,10$ & Mycobacterium spp & $1,2,3,4,6$ & M. simiae \\
\hline M. smegmatis & 1 & $1,2,3,10$ & Mycobacterium spp & $1,2,3,6,14$ & M. smegmatis \\
\hline
\end{tabular}

Abreviaturas: No = Números de cepas; $\mathrm{NR}=$ No realizado.

En la tabla 2 se presentan los resultados obtenidos de la identificación de los 73 aislados clínicos de MNT mediante la prueba molecular GenoType Mycobacterium CM. Un total de 65 aislados se identificaron a nivel de especies, predominando M. intracellulare 21 (28.8\%) dentro del complejo MAI y M. fortuitum 22 (30.1\%), mientras que en ocho aislados sólo se pudo confirmar que pertenecían al género Mycobacterium.

En la tabla 3 se muestran los resultados de la identificación a nivel de especie por la técnica GenoType Mycobacterium AS de los ocho aislados de MNT no identificados con el sistema GenoType Mycobacterium CM, al aplicar esta técnica se lograron identificar sólo cuatro aislados como pertenecientes a M. simiae.

En la tabla 4 se muestran los resultados obtenidos al comparar las pruebas bioquímicas frente a las técnicas moleculares GenoType Mycobacterium CM y GenoType Mycobacterium AS.

Estos resultados mostraron una coincidencia en 63 de los aislados analizados, en 6 aislados no se obtuvo coincidencia entre ambas técnicas, y en los 4 restantes sólo fueron identificados a nivel de género por los dos ensayos moleculares.

Para el cálculo de la concordancia entre la identificación bioquímica y las pruebas moleculares no se tuvieron en cuenta los cuatro aislados que sólo fueron identificados hasta género Mycobacterium empleando los sistemas comerciales GenoType Mycobacterium CM y GenoType Mycobacterium AS marcados con una (b). Por tanto, el número total de aislados que pudieron ser identificados y comparados se consideró 69 obteniendo una concordancia del 91.3\%.

\section{DISCUSIÓN}

La técnica GenoType Mycobacterium CM permite diferenciar las 24 especies micobacterianas que se asocian a micobacteriosis con más frecuencia. Todas las cepas de referencia utilizadas en esta investigación fueron identificadas de manera adecuada, observándose los correspondientes patrones de hibridación específicos de cada una de ellas. Esto corrobora la calidad de este sistema comercial, lo cual ha sido ya publicado por otros autores. ${ }^{12,13}$ Para la identificación de M. fortuitum como especie con el sistema GenoType Mycobacterium CM se emplearon cuatro cepas
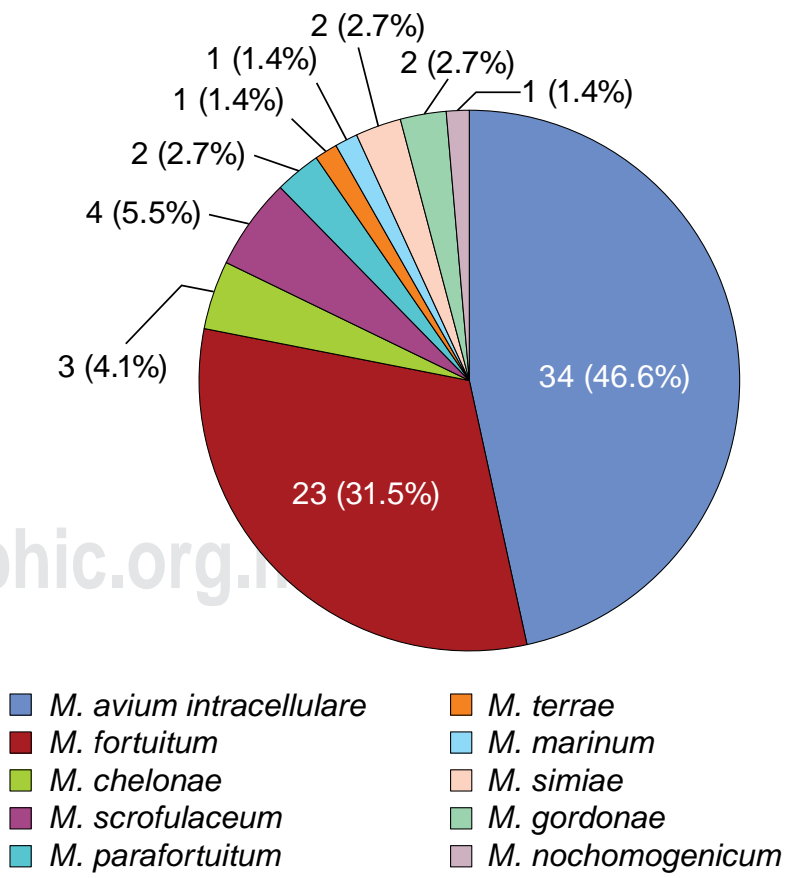

Figura 1: Frecuencia de especies de micobacterias no tuberculosas (MNT) identificadas por la prueba convencional en 73 aislados clínicos procedentes de instituciones de salud de Cuba. Abril 2017-diciembre 2018. 
de referencia dentro de las 12 utilizadas en este estudio, pues en la tabla de interpretación del Manual de Instrucciones del ensayo indica que existen dos patrones de bandas diferentes para esta especie. Esto debe estar motivado por la elevada variabilidad genética de esta especie. No obstante, en este trabajo las cuatro cepas de $M$. fortuitum mostraron el mismo patrón $(1,2,3,7,14){ }^{8}$

Como se puede observar en la tabla 1 las cepas de referencia pertenecientes a las especies $M$. simiae, $M$. smegmatis y M. phlei se identificaron correctamente con el empleo del ensayo GenoType Mycobacterium AS, el cual es capaz de identificar especies de micobacterias que con menor frecuencia son responsables de infecciones en el hombre. ${ }^{9}$

La identificación convencional de MNT se lleva a cabo mediante pruebas que permiten detectar características fenotípicas y bioquímicas. Estas pruebas son muy utilizadas, pero presentan importantes limitaciones las cuales contribuyen al problema de la diferenciación de especie y del manejo clínico de estas infecciones. Las pruebas bioquímicas en ocasiones presentan variabilidad, ya que la expresión de un fenotipo determinado depende de condiciones ambien-

Tabla 2: Resultados de la identificación molecular mediante el ensayo GenoType Mycobacterium CM de 73 aislados clínicos de micobacterias no tuberculosas (MNT) procedentes de instituciones de salud de Cuba. Abril 2017-diciembre 2018.

\begin{tabular}{|l|c|c|c|}
\hline $\begin{array}{l}\text { GenoType } \\
\text { Mycobacterium CM }\end{array}$ & $\mathbf{n}$ & $\%$ & $\begin{array}{c}\text { Patrón de } \\
\text { bandas }\end{array}$ \\
\hline M. avium spp & 12 & 16.4 & $1,2,3,4$ \\
\hline M. intracellulare & 21 & 28.8 & $1,2,3,9$ \\
\hline M. fortuitum & 22 & 30.1 & $1,2,3,7,14$ \\
\hline M. chelonae & 2 & 2.7 & $1,2,3,5,10$ \\
\hline M. gordonae & 2 & 2.7 & $1,2,3,8,10$ \\
\hline M. scrofulaceum & 3 & 4.1 & $1,2,3,9,10$ \\
\hline M. kansasii & 1 & 1.4 & $1,2,3,10,12$ \\
\hline M. abscessus & 2 & 2.7 & $1,2,3,5,6,10$ \\
\hline Mycobacterium spp & 8 & 11.0 & $1,2,3,10$ \\
\hline
\end{tabular}

Abreviaturas: $\mathrm{n}=$ Número de aislados.

Tabla 3: Resultados de la identificación por la prueba GenoType Mycobacterium AS de los ocho aislados de micobacterias no tuberculosas (MNT) no identificados con GenoType Mycobacterium CM.

\begin{tabular}{|l|c|c|c|}
\hline $\begin{array}{l}\text { GenoType } \\
\text { Mycobacterium AS }\end{array}$ & $\mathbf{n}$ & $\%$ & $\begin{array}{c}\text { Patrón de } \\
\text { bandas }\end{array}$ \\
\hline M. simiae & 4 & 5.5 & $1,2,3,4,6$ \\
\hline Mycobacterium spp & 4 & 5.5 & $1,2,3,12$ \\
\hline
\end{tabular}

Tabla 4: Comparación entre el método convencional y los métodos moleculares para la identificación de micobacterias no tuberculosas (MNT) en 73 aislados.

\begin{tabular}{|c|c|c|}
\hline $\begin{array}{l}\text { Identificación } \\
\text { pruebas } \\
\text { bioquímicas }\end{array}$ & $\begin{array}{c}\text { Identificación genotípica } \\
\text { Mycobacterium } \\
\text { GenoType CM y AS }\end{array}$ & $\begin{array}{l}\text { No de } \\
\text { aislados }\end{array}$ \\
\hline \multirow[t]{4}{*}{ Complejo MAI } & M. avium & 12 \\
\hline & M. intracellulare & 20 \\
\hline & M. simiae & $1^{a}$ \\
\hline & Mycobacterium spp & $1^{\mathrm{b}}$ \\
\hline \multirow[t]{2}{*}{ M. fortuitum } & M. fortuitum & 22 \\
\hline & M. abscessus & $1^{a}$ \\
\hline \multirow[t]{2}{*}{ M. chelonae } & M. chelonae & 2 \\
\hline & M. abscessus & $1^{a}$ \\
\hline M. gordonae & M. gordonae & 2 \\
\hline M. kansasii & M. kansasii & 1 \\
\hline \multirow[t]{2}{*}{ M. scrofulaceum } & M. scrofulaceum & 3 \\
\hline & M. simiae & $1^{a}$ \\
\hline M. simiae & M. simiae & 2 \\
\hline M. terrae & M. intracellulare & $1^{a}$ \\
\hline M. marinum & M. kansasii & $1^{a}$ \\
\hline M. nochomogenicum & Mycobacterium spp & $1^{b}$ \\
\hline M. parafortuitum & Mycobacterium spp & $2^{b}$ \\
\hline
\end{tabular}

Abreviaturas: ${ }^{a}$ Resultados discordantes entre ambos métodos.

${ }^{b}$ Por pruebas moleculares solo se identificó el género.

tales. La metodología puede arrojar diferentes resultados al modificarse ciertos factores, como la preparación de los medios y reactivos, cantidad y tiempo de crecimiento de la cepa a identificar, e incluso el observador, todo ello dificulta la interpretación de los resultados obtenidos. ${ }^{14}$

Los resultados obtenidos en este trabajo son superiores a lo reportado por Castillo y colaboradores (2013) para determinar la frecuencia de micobacteriosis pulmonar en pacientes con infección por VIH atendidos en el IPK, donde se registraron como especies más predominantes a $M$. fortuitum con $18.91 \%$ y complejo MAI con $16.21 \%$. Los resultados de esta investigación son similares a los reportados por Mederos y colegas (2014), donde se analizaron aislados de MNT de muestras clínicas y detectaron al complejo MAI (43\%) entre las especies más frecuentes. ${ }^{15,16}$

Otras investigaciones realizadas en América Latina reportan resultados similares, como el estudio publicado por Godoy y colaboradores en Venezuela, que identificó un mayor predominio del complejo MAI (21.7\%) seguido por M. fortuitum (17.4\%). Sin embargo, los resultados obtenidos difieren de los reportados en otra región por 
Neumol Cir Torax. 2019; 78 (3): 290-295

Nasiri y colegas (2017), quienes en Irán obtuvieron mayor porcentaje de aislados de M. simiae (38.7\%) y M. fortuitum (19.3\%), siendo el complejo MAI (8\%) de menos predominio. En algunos países del Medio Oriente, se ha registrado la presencia de M. simiae como una de las especies de MNT más prevalentes, lo cual pudiera estar relacionado con las características del área geográfica evaluada.6,17

En Cuba, es la primera vez que se utilizan los ensayos GenoType Mycobacterium CM y GenoType Mycobacterium AS para la identificación de MNT. Con estas técnicas se logró determinar la especie en el $91.3 \%$ de los aislados clínicos estudiados debido a que estos ensayos no permiten identificar las especies M. nochomogenicum y M. parafortuitum.

Recién y motivado fundamentalmente por la disminución de la tuberculosis en los países desarrollados, y el incremento de las MNT en múltiples procesos patológicos, tanto en pacientes inmunocompetentes como inmunocomprometidos, se han incrementado los estudios de identificación y caracterización de MNT de importancia clínica. Hoy, se reconocen más de 150 especies, muchas de estas descritas en las últimas dos décadas gracias a los métodos moleculares. ${ }^{1}$

Los aislados de $M$. simiae en esta investigación son significativos. Esta especie fue reportada inicialmente por Karasova en 1965 en aislados de muestras de monos importados por Hungría desde India, que dieron la prueba de la tuberculina negativa. En Cuba, Valdivia y colaboradores (1971), describieron una micobacteria que está relacionada bioquímicamente con el serotipo 1 de $M$. simiae que fue denominada M. habana. ${ }^{18}$

Los resultados obtenidos en esta investigación son superiores a los reportados por Singh y colegas en 2013, en la India. Las especies de MNT más comúnmente encontradas al utilizar las técnicas moleculares Genotype Mycobacterium CM/AS fueron; M. fortuitum 20 (33.3\%), M. intracellulare $11(18.3 \%)$ y M. avium 4 (6.7\%)..$^{13}$

En relación con el grado de dificultad, si bien ambos métodos necesitan un adiestramiento previo, las pruebas bioquímicas son más laboriosas y requieren de una inversión de tiempo considerable porque deben ser observadas prácticamente de forma diaria por varias semanas, además de lo engorroso de trabajar con material biológico por mucho tiempo, se complica más porque cada laboratorio conforma diversos protocolos de identificación bioquímica de acuerdo a la disponibilidad. Los sistemas GenoType Mycobacterium CM y GenoType Mycobacterium AS requieren de 2-3 días de trabajo y sólo se mantiene contacto con material infeccioso durante la extracción de ADN. La técnica utiliza menor número de soluciones, las mismas se pueden preparar con anterioridad en grandes volúmenes, y almacenarse por tiempo prolongado.

Los resultados obtenidos en este estudio fueron similares a los reportados por Richter y colaboradores (2006) en un estudio realizado en Alemania donde registraron valores de concordancia de 92\% para los sistemas GenoType Mycobacterium CM y GenoType Mycobacterium AS. ${ }^{19}$ Sin embargo, fueron superiores a lo reportado por Gittiy y su equipo en una investigación en Grecia (2006) donde obtuvieron una concordancia de $84.2 \% .{ }^{20}$

Durante el desarrollo de este trabajo se pudieron constatar en la práctica las ventajas de la aplicación de las técnicas GenoType Mycobacterium CM y GenoType Mycobacterium AS frente al método convencional. Algunas de estas ventajas incluyen el poco tiempo requerido para la obtención de resultados, la relativa facilidad de realización e interpretación de la prueba, y la limitada manipulación de material infeccioso que se traduce en disminución de riesgo biológico.

Las pruebas GenoType Mycobacterium CM y GenoType Mycobacterium AS son herramientas adecuadas (rápidas, sensibles, operativas) para la identificación de las especies de MNT. Los resultados obtenidos en este estudio demostraron que con la introducción de estas técnicas moleculares se ha logrado un verdadero avance en el acortamiento del tiempo para diagnóstico, permitiendo así una pronta implementación del tratamiento adecuado para los pacientes afectados.

\section{REFERENCIAS}

1. Tortoli E. Microbiological features and clinical relevance of new species of the genus Mycobacterium. Clin Microbiol Rev 2014; 27(4):727-752. doi: 10.1128/CMR.00035-14.

2. Hoefsloot W, van Ingen J, Andrejak C, et al. Nontuberculous Mycobacteria Network European Trials Group. The geographic diversity of nontuberculous mycobacteria isolated from pulmonary samples: An NTM-NET collaborative study. Eur Respir J 2013;42(6):1604-1613. doi: 10.1183/09031936.00149212.

3. Mederos LM, Fleites G, Capó V, De Armas Y, et al. Infección diseminada por Mycobacterium szulgai con compromiso pulmonar y ganglionar en un paciente con sida. SIIC 2012; 18(8):776-780.

4. Mederos CLM, Reyes PA, Valdes AL, et al. Coinfección por Mycobacterium malmoense y Mycobacterium tuberculosis en paciente con el síndrome de inmunodeficiencia humana. Rev Peru Med Exp Salud Pública 2014;31(4):788-792.

5. Mederos LM, Fleites G, Acosta MA, et al. Micobacteriosis peritoneal porMycobacterium avium-intracellulare en paciente con el síndrome de inmunodeficiencia adquirida. SIIC 2017; 22:352-356.

6 Godoy MJ, Orozco L, Hernández C, et al. Identificación de micobacterias no tuberculosas: comparación de métodos bioquímicos y moleculares. Rev Soc Ven Microbiol 2008;28(2):1315-2556.

7. Leiva CT. Evaluación de una técnica de hibridación reversa para identificación rápida de micobacterias en Chile. Rev Chil Enf Respir 2012; 28:9-15. doi.org/10.4067/S0717-73482012000100002.

8. GenoType Mycobacterium CM VER 2.0. Fecha de acceso: diciembre 2018. Disponible en: www.hain-lifescience.de.

9. GenoType Mycobacterium AS VER 1.0. Fecha de acceso: diciembre 2018. Disponible en: www.hain-lifescience.de. 
10. Runyon EH. Anonymous mycobacteria in pulmonary disease. Med Clin North Am 1959;43(1):273-290.

11. Fernández de Vega FA, Moreno JE, González Martín J, et al. Micobacterias. En: Cercenado E, Cantón R, editores. Procedimientos en microbiología clínica. España: editorial; 2005.p.12-39.

12. Lee AS, Jelfs P, Sintchenko V, Gilbert LG. Identification of nontuberculous mycobacteria: utility of the GenoType Mycobacterium CM/ AS assay compared with HPLC and 16S rRNA gene sequencing. J Med Microbiol 2009;58(Pt 7):900-904. doi: 10.1099/jmm.0.007484-0.

13. Singh AK, Maurya AK, Umrao J, et al. Role of GenoType (®) Mycobacterium Common Mycobacteria/Additional Species Assay for Rapid Differentiation Between Mycobacterium tuberculosis Complex and Different Species of Non-Tuberculous Mycobacteria. J Lab Physicians 2013;5(2):83-89. doi: 10.4103/0974-2727.119847.

14. Springer B, Stockman L, Teschner K, Roberts GD, Böttger EC. Two-laboratory collaborative study on identification of mycobacteria: molecular versus phenotypic methods. J Clin Microbiol 1996;34(2):296-303.

15. Castillo A, Díaz M, Mederos LM, et al. Infección pulmonar por micobacterias ambientales en pacientes VIH/SIDA atendidos en el Instituto de Medicina Tropical Pedro Kourí, enero 2006-junio 2008. Rev Panam Infectol 2013;15(1-4):33-40.
16. Mederos CLM, Martínez RMR, Sardiñas AM, et al. Utilidad del cultivo rápido en medio líquido Bact/Alert 3D en el diagnóstico micobacteriano de muestras clínicas. AVFT 2016;35(3):77-82.

17. Nasiri MJ, Dabiri H, Fooladi AAl, Amini S, Hamzehloo G, Feizabadi MM. High rates of nontuberculous mycobacteria isolation from patients with presumptive tuberculosis in Iran. New Microbes New Infect 2017; 21:12-18. doi: 10.1016/j.nmni.2017.08.008.

18. Valdivia JA, Suárez MR, Echemendía MA. Mycobacterium habana: probable nueva especie dentro de las micobacterias no clasificadas. Bol Hig Epid 1971;9:65-73.

19. Richter E, Rüsch-Gerdes S, Hillemann D. Evaluation of the GenoType Mycobacterium Assay for identification of mycobacterial species from cultures. J Clin Microbiol 2006; 44(5):1769-1775.

20. Gitti Z, Neonakis I, Fanti G, Kontos F, Maraki S, Tselentis Y. Use of the GenoType Mycobacterium CM and AS assays to analyze 76 nontuberculous mycobacterial isolates from Greece. J Clin Microbiol 2006;44(6):2244-2246.

Conflicto de intereses: Los autores declaran no tener conflicto de intereses. 